\title{
Incidencia de diarrea infantil en una guardería.
}

\section{Comunicación Breve}

Jorge Alonzo-Salomón ${ }^{1}$, Oneila Roque-Rodríguez².

${ }^{1}$ Coordinación de Ingeniería Ambiental. ${ }^{2}$ Programa de la Maestría en Ingeniería Ambiental, Facultad de Ingeniería, Universidad Autónoma de Yucatán, Mérida, Yucatán, México.

\section{RESUMEN.}

Introducción. Las guarderías agrupan niños en ambientes con mayor exposición a agentes causantes de enfermedad que el hogar, resultando en mayores tasas de incidencia de diarrea (TID) infantil y con las enfermedades diarreicas y respiratorias como principales causas de morbilidad. Este trabajo describe el comportamiento de (TID) en infantes de 1.5-23 meses de edad en una guardería de Mérida, Yucatán, México.

Materiales y métodos. Observamos prospectivamente a todos los infantes de 1.5-23 meses de edad que ingresaron a una guardería de Mérida desde agosto de 1996 hasta julio de 1997. Los dividimos en 4 grupos de edad para obtener el número de episodios de diarrea, el tiempo en riesgo por niño por día, derivar las TID y efectuar comparaciones.
Resultados. La TID total $=2.7$ episodios $/$ niñoaño [intervalo de confianza del 95\% (IC 95\%) = 2.2-3.2] La TID para los menores de un año $=5.2$ episodios/niño-año (IC 95\%= 4.1-6.3) que es 4 veces la TID del grupo mayores de un año de edad [razón de tasas de incidencia de diarrea (RTID) = 4.1; IC 95\% = 2.9-5.6] Para el grupo 1.5-5 meses la $\mathrm{TID}=7.0$ episodio $/$ niño-año $(\mathrm{IC} 95 \%=4.9$ 9.1) la mayor TID, que es 10 veces la TID del grupo con la menor TID, el de 18-23 meses de edad.

Conclusiones. Las mayores TID en la guardería se presentan entre los menores de un año, siendo el grupo infantil de menores de 6 meses de edad el más afectado. (Rev Biomed 2001; 12:256-261)

Palabras clave: Diarrea infantil, guarderías, epidemiología, Yucatán México.

Solicitud de sobretiros: Jorge E. Alonzo-Salomón, Coordinación de Ingeniería Ambiental, Facultad de Ingeniería, Universidad Autónoma de Yucatán. Apartado Postal 1-150 Cordemex, C.P. 97111, Mérida, Yucatán, México.Email: asalomon@tunku.uady.mx 
J Alonzo-Salomón, O Roque-Rodríguez.

\section{SUMMARY.}

Incidence of diarrhea in a day care center. Background. Day-care centers gather children together in an environment with higher exposure to agents causing diseases than those encountered in their home environment, resulting in higher incidence rates of diarrhea (IRD) in the day-care center than at home, and with the diarrhoeal and respiratory diseases as main causes of morbidity. This study describes the behavior of the incidence rate of diarrhea (IRD) in infants 1.5-23 months of age in a day-care center in Mérida, Yucatan, Mexico.

Material and methods. We prospectively observed all the infants 1.5-23 months of age who attended a day-care center in Merida City from August 1996 to July 1997. We divided them in 4 age groups in order to obtain the number of diarrhea episodes, and the time at risk of diarrhea per child per day, to derive the IRD, and to make comparisons among the groups.

Results. The total IRD $=2.7$ episodes / child-year [95\% confidence interval $(95 \% \mathrm{CI})=2.2-3.1]$ The IRD for children less than one year of age $=5.2$ episodes/child-year [95\% confidence interval $(95 \% \mathrm{CI})=4.1-6.3]$ this IRD is 4 times the IRD for infants older than one year of age [incidence rate ratio of diarrhea $(\mathrm{IRRD})=4.1,95 \% \mathrm{CI}=2.9$ 5.6] The IRD for infants $1.5-5$ months of age $=$ 7.0 episodes / child-year $(95 \% \mathrm{CI}=4.9-9.1)$ the highest IRD among groups, and is 10 times greater the IRD of infants 18-23 months of age, the group with the lowest IRD.

Discussion. The highest IRD in the day-care center was among infants less than 1 year old, and the group of infants less than 6 months old is the most affected. (Rev Biomed 2001; 12:256-261)

Key words: Day-care center, child diarrhea, epidemiology, Yucatan Mexico.

\section{INTRODUCCIÓN.}

El presente trabajo es un estudio descriptivo sobre la ocurrencia de la tasa de incidencia de diarrea TID en infantes de una guardería de la ciudad de Mérida, Yucatán, México. Nuestro interés al desarrollar este trabajo, es el proporcionar información cuantitativa sobre el comportamiento de la TID en el marco de una guardería local.

En la actualidad las guarderías han adquirido importancia al ser estos centros una alternativa para el cuidado de hijos de madres trabajadoras que por no poder atenderlos en el hogar han decidido utilizar los servicios de las guarderías para su cuidado. Lo anterior se relaciona con un incremento de madres en la fuerza de trabajo, con un mayor número de guarderías que prestan servicio a la sociedad y con un aumento en el número de niños que son atendidos en las guarderías (1-3) La utilización de las guarderías como centros de cuidado originan el agrupamiento de infantes y preescolares en ambientes diferentes a los encontrados en el hogar, favoreciéndose las condiciones para que estos niños se vean expuestos a nuevos agentes causantes de enfermedades $(4,5)$. Entre estas enfermedades las diarreicas y las respiratorias son las principales causas de morbilidad en guarderías (6-8)

La metodología utilizada en este estudio para describir la ocurrencia de las enfermedades diarreicas en la guardería se fundamenta en la aplicación de un estudio observacional de cohortes prospectivo; de este estudio, observamos una población dinámica de infantes de 1.5-23 meses de edad y como resultado obtuvimos los datos para derivar la TID. La tasa de incidencia, que derivamos y utilizamos durante todo nuestro estudio, es la versión conocida como la tasa de densidad de incidencia (9-13)

\section{MATERIAL Y MÉTODOS.}

Tipo de Estudio. El diseño epidemiológico que utilizamos en esta investigación fue un estudio observacional de cohortes prospectivo (9-12) la cohorte de estudio se integró con una población dinámica de infantes de 1.5 a 23 meses de edad,

\section{Revista Biomédica}


los cuales se observaron con el propósito de conocer para cada uno de ellos, su historia individual de seguimiento, es decir, el número de episodios de diarrea por niño por día y el tiempo en riesgo de diarrea por niño por día. La duración de la investigación fue del 1 agosto de 1996 al 31 de julio de 1997.

Población Estudiada. El diseño del estudio de cohortes seleccionado implicó el seguimiento de una población dinámica de infantes de 1.5-23 meses de edad admitidos en la guardería desde el primero de agosto de 1996. Iniciamos el seguimiento de la cohorte con todos los niños inscritos como alumnos regulares y con un registro sobre la ocurrencia de diarrea y sus asistencias a la guardería por niño por día. En nuestro estudio no examinamos directamente a los infantes que integraron la cohorte de estudio, sino que nos encargamos de observar y registrar la evolución diaria del estado de salud y las asistencias de los infantes a la guardería; dicha evolución diaria la observamos de los archivos médicos, bitácoras de enfermeras y listas de asistencia de las maestras de la guardería. Las entradas y salidas de la cohorte se realizaron durante todo el año, lo cual se encuentra registrado en la historia individual de seguimiento de cada infante. La población estudiada se dividió en 4 grupos de edad: $1.5-5,6-11,12-17$ y $18-23$ meses de edad coincidiendo nuestros grupos de estudio con la clasificación por grupo de edad que la guardería utiliza para integrar a sus alumnos por salones de clase.

Criterio de inclusión. Para ser incluidos en la población estudiada, los infantes debían cumplir los siguiente criterios: estar inscrito como alumno regular de la guardería, tener entre 1.5 y 23 meses de edad al momento del inicio del estudio y estar libre de alguna enfermedad o padecimiento crónico, según el criterio del médico de la guardería.

Definición de Variables. Diarrea. La deposición de heces líquidas por un niño con una frecuencia no usual, siendo esto determinado por el médico o enfermera de la guardería. Episodio de diarrea. La ocurrencia de diarrea en un niño separada de una diarrea previa en por lo menos 7 días. Niñodía. Cualquier día de lunes a viernes en el cual un infante de 1.5 a 23 meses de edad, regularmente inscrito, asiste a la guardería por más de 1 hora. Tiempo en riesgo. Suma de los períodos de niñodía correspondiente a la población infantil estudiada (11-13). Tasa de incidencia de diarrea. El número de episodios de diarrea registrados durante un intervalo de tiempo dividido entre la sumatoria niño-día en el mismo intervalo de tiempo (11-13); para efectos del cálculo de la tasa de incidencia anual se definió un año como equivalente a 200 días hábiles.

Recolección de datos. Para obtener la información necesaria para formar la historia de seguimiento de cada infante, realizamos visitas semanales a la guardería con el objeto de examinar los expedientes médicos, las bitácoras de las enfermeras, las listas de asistencia y a su vez, mediante entrevistas con el personal involucrado en la generación de dicha información, registrar la información que necesitábamos para obtener del número de episodios de diarrea por niño por día y el tiempo en riesgo de diarrea por niño por día; la información recolectada fue ordenada en una base de datos y se resumió en una tabla de contingencia de donde se derivaron las tasas de incidencia de diarrea. La información que obtuvimos nos fue proporcionada por el médico, las maestras encargadas de los niños y la enfermera de la guardería y se realizó con la autorización de la dirección de la guardería. Se generaron los propios datos realizando previamente, un análisis de las fuentes de información, para ver la correspondencia entre los informes de los casos de niños con diarrea, sus asistencias a la guardería y la generación de nuestros datos.

Procesamiento de datos y análisis estadístico.

Los datos obtenidos fueron resumidos utilizando tablas de contingencia, de donde se derivo la TID y la razón de tasas de incidencia de

Vol. 12/No. 4/Octubre-Diciembre, 2001 
J Alonzo-Salomón, O Roque-Rodríguez.

Cuadro 1

Incidencia de diarrea por grupo de edad.

\begin{tabular}{|c|c|c|c|c|}
\hline $\begin{array}{l}\text { Grupo de edad } \\
\quad \text { (meses) }\end{array}$ & Episodios & Niño-día & Incidencia & (IC 95\%) \\
\hline $1.5-5$ & 43 & 1230 & 7.0 & $(4.9-9.1)$ \\
\hline $6-11$ & 40 & 1984 & 4.0 & $(2.8-5.3)$ \\
\hline $12-17$ & 24 & 2463 & 1.9 & $(1.2-2.7)$ \\
\hline $18-23$ & 11 & 3146 & 0.7 & $(0.3-1.1)$ \\
\hline TOTAL & 118 & 8823 & 2.7 & $(2.2-3.2)$ \\
\hline
\end{tabular}

Episodios / niño-año.

IC $=$ intervalo de confianza

diarrea (RTID) $(12,13)$. Para la TID se derivaron los intervalos de confianza del $95 \%$ y para la RTID se efectuaron pruebas de hipótesis utilizando la aproximación normal a la distribución binomial; el cálculo de los intervalos de confianza del 95\% se efectuó utilizando el método de prueba de Miettinen (12,13).

\section{RESULTADOS.}

Observamos un total de 151 infantes de 1.523 meses de edad, que ingresaron a la cohorte de estudio durante el año que duró la investigación. Como resultado del análisis de la historia de seguimiento de cada uno de ellos, obtuvimos los datos que se resumen en el cuadro $1 \mathrm{y}$ del análisis del mismo se obtuvo que la TID en el grupo de menores de un año es 5.2 episodios por niño por año (IC 95\% 4.1-6.3) la TID en los infantes mayores de un año es 1.2 episodios por niño por año (IC 95\% 0.8-1.7) Comparando la TID de los menores de un año de edad con respecto a la TID de los mayores de un año se obtiene una RTID de 4.1 (IC95\% 2.9-5.6) La TID en el grupo de menores de 6 meses es 7.0 episodios por niño por año (IC 95\% 4.9-9.1) esta tasa es la de mayor magnitud por grupo de edad en la guardería, misma que es 10 veces el valor de la TID del grupo de 18-23 meses, que es la menor tasa observada por grupo de edad.

Revista Biomédica

\section{DISCUSIÓN.}

Comparaciones internas en nuestro estudio con relación al comportamiento de la TID por grupo de edad, muestran que la TID se incrementa uniformemente al pasar de un grupo de menor a uno de mayor edad, este patrón de comportamiento de la TID es similar al obtenido en otros estudios (14-17). Comparaciones con relación a valores específicos de la TID por grupo edad, muestran que la TID para el total de infantes en nuestro estudio es igual a la TID de 1991 para niños menores de 2 años de guarderías de Houston, Estados Unidos (EU) después de estar un mes enrolados en la guardería y es aproximadamente igual a la TID de 1992 para niños mayores de 2 años de guarderías de Carolina del Norte, EU (15), Con respecto a comparaciones con otras TID obtenidas de estudios realizados en países subdesarrollados, se observa que la TID total de nuestro estudio es 2.7 veces menor que la de 1992 para niños menores de 2 años de edad de guarderías de Colombia (16) y un $12.5 \%$ mayor que la tasa de 1995 para niños de 1 hasta 3.5 años de edad de guarderías de Ecuador (17). Por grupo semestral de edad, comparando nuestros resultados con las TID de 1991 de guarderías de Houston, EU se observa que en el grupo de menores de 6 meses de edad, la TID de en nuestro estudio es el doble de la obtenida en infantes de la misma edad de las 
Incidencia de diarrea infantil.

guarderías de Houston; para los infantes de 6-11 meses de edad, es un $11 \%$ mayor; para los de 1217 meses, es un 35\% menor y para los de 18-23 meses, la tasa de nuestro estudio es un $46 \%$ menor que la tasa de los niños de Houston.

De las comparaciones efectuadas se puede decir que el patrón observado sobre el comportamiento de la TID es, en general, tener las mayores tasas en los grupos de menor edad, en el caso particular de nuestro estudio, en el de menores de un año y con el grupo de menores de 6 meses como el más afectado. Las posibles razones para este comportamiento en la TID pueden estar dados por la variabilidad de la respuesta inmune con la edad (19). Las diferencias con respecto a la magnitud entre las TID observadas, podrían explicarse por la variabilidad en las condiciones ambientales e higiénicas dentro de las guarderías (20,21-23) De las comparaciones efectuadas, también podemos decir, que el patrón observado sobre el comportamiento de la TID en nuestro estudio es el esperado, es decir, que la mayores TID observadas correspondes al grupo de menores de 6 y al de 6-11 meses de edad, observándose la mayor magnitud en de la TID en los menores de 6 meses.

Las principales limitaciones de nuestro trabajo son con respecto a la comparabilidad entre las magnitudes que obtuvimos para las TID con respecto a las obtenidas en otros estudios. Estas limitaciones se refieren a que en nuestro trabajo sólo consideramos una guardería como universo de estudio, por lo que no podemos tener una medida de la variabilidad de la TID entre guarderías. Una forma de evitarlo, consiste en tomar una muestra aleatoria de niños de guarderías seleccionadas al azar y tener como unidad de análisis la variable niño-día o la guardería. También otro factor que oscurezca la comparabilidad de nuestros resultados es la falta de información sobre variables, tales como las condiciones socioeconómicas de los niños involucrados en el estudio o bien alguna característica socioeconómica de la guardería estudiada.

Dado que las enfermedades diarreicas continúan siendo una causa importante de enfermedad entre niños asistentes a guarderías sería importante conocer más sobre su comportamiento y distribución considerando una muestra representativa de guarderías de la localidad.

\section{REFERENCIAS.}

1.- Peth R. The NICHD Study of Early Child Care. Public Information and Communication Branch of the National Institute of Child and Human Development. Documento disponible en: URL: http:// www.nichd.nih.gov/publications/pubs/ early_child_care.htm

2.- Baqui, AH, Black RE, Sack RB, Yunus MD, Siddique AK. Infectious diarrhoea in children: controlling transmission in the child care setting. J Paediatr Child Health 1994; 30:201-9.

3.- Holmes SJ, Morrow AL, Pickering LK. Child-care practices: effects of social change on the epidemiology of infectious diseases and antibiotic resistance. Epidemiol Rev 1996; 18:10-28.

4.- Reves RR, Morrow AL, Bartlett AV, Caruso CJ, Plumb RL, Bening T, et al. Child day care increases the risk of clinic visits for acute diarrhea and diarrhea due to rotavirus. Am J Epidemiol 1993; 137:97-107.

5.- Churchill RB, Pickering LK. Infection control challenges in child-care centers. Infect Dis Clin North Am 1997; 11:347-65.

6.- Wald ER, Dashefsky B, Byers C, Guerra N, Taylor F. Frequency and severity of infections in day care. $\mathrm{J}$ Pediatr 1988; 112:540-6.

7.- Bartlett AV, Moore M, Gary GW, Karen M S, Erben JJ, Betty AM. Diarrheal illness among infants and toddlers in day care centers. I Epidemiology and pathogens. Pediatrics 1985; 107:495-502.

8.- Pickering LK, Evans DG, DuPont HL, Volled JJI, Evans DJJ. Diarrhea caused by Shigella, Rotavirus, and Giardia in day-care centers: prospective study. J Pediatr 1981; 99:51-6. 


\section{J Alonzo-Salomón, O Roque-Rodríguez.}

9.- $\quad$ Samet MJ, Muñoz A. Cohort Studies. Epidemiol Rev 1998; 20: 1-28.

10.- Armitage P, Colton T, editors. Encyclopedia of Biostatistics. New York: John Wiley; 1998.

11.- Hernberg S. Introduction to Occupational Epidemiology. Boca Raton: CRC Press; 1992.

12.- Kleinbaum DG, Kupper LL, Morgenstern H. Epidemiologic Research. New York: Van Nostrand Reinhold; 1982.

13.- Rothman KJ. Modern Epidemiology. Boston: Little Brown; 1986.

14.- Sullivan P, Williams MS, Woodward MD, Pickering MD, DuPont HL. Longitudinal study of the occurrence of diarrheal diseases in day care centers. Am J Public Health 1984; 74:987-91.

15.- Kotch JB, Weigle KA, Weber JD, Clifford RM, Harms TO, Loda FA, et al. Evaluation of Hygienic Intervention in Child day- care centers. Pediatrics 1992; 93 (suppl): 991-4.

16.- Hillis SD, Miranda CM, McCann M, Bender D, Weigle K. Day care attendance and diarrheal morbidity in Colombia. Pediatrics 1992; 90:582-8.

17.- Sempertegui F, Estrella B, Egas J, Carrion P. Risk of diarrheal disease in Ecuatorian day-care centers. Pediatric Infect Dis J 1995; 14:606-12.

18.- Staad MA, Morrow AL, Reves RR, Bartlett AV, Pickering LK. Diarrhea in children newly enrolled in daycare centers in Houston. Pediatr Infect Dis J 1991; 10:2826

19.- Yang KD, Hill HR. Immune responses to infectious diseases: an evolutionary perspective. Pediatr Infect Dis J 1996; 15:355-64.

20.- Van R, Wun CC, Morrow AL, Pickering LK. The effect of diaper type and overclothing on fecal contamination in day-care centers. JAMA 1990; 265:1840-4.

21.- Van R, Morrow AL, Reves RR, Pickering LA. Environmental contamination in child day-care centers. Am J Epidemiol 1991; 133:460-70.

22.- Weniger BG, Ruttenberg AJ, Goodman RA, Juranek

\section{Revista Biomédica}

DD, Wahlquist SP, Smith D. Fecal coliforms on environmental surfaces in two day care centers. Appl Environ Microbiol 1983; 45:733-5.

23.- Ekanem EE, DuPont HL, Pickering LK, Selwyn B, Hawkins CM. Transmission dynamics in day-care centers. Am J Epidemiol 1983; 118:562-73. 